\title{
On upcrossing inequalities for subadditive superstationary processes
}

\author{
MICHAEL KRAWCZAK \\ Institut für Mathematische Stochastik, Lotzestr. 13, D-3400 Göttingen, West Germany
}

(Received 29 September 1984)

\begin{abstract}
Bishop [2] has given a proof of Birkhoff's ergodic theorem by establishing upcrossing inequalities similar to those of Doob. Such inequalities can be considered as quantitative improvements of convergence theorems: while convergence a.e. means that the number of upcrossings of any interval is a.e. finite, they assert integrability and prove bounds for the integrals. The main point of this paper is to prove upcrossing inequalities for the class of subadditive superstationary processes introduced by Abid [1] as a common generalization of Kingman's [5] subadditive stationary processes and Krengel's [6] superstationary processes. We make use of ideas of Smeltzer [7] who handled the subadditive stationary discrete parameter case in his unpublished thesis. In the continuous parameter case our upcrossing inequality requires more restrictive conditions than the corresponding convergence theorem, due to Hachem [3]. We actually show by example that the number of upcrossings need not be integrable under the assumptions of Hachem even for additive stationary processes.
\end{abstract}

Let $\mathcal{N}=\{(s, t): 0 \leq s<t, s, t \in \mathbb{Z}\}$ and consider the space $\mathbb{R}^{\mathbf{Z}^{2}}$. If $\pi_{s, t}$ denotes the projection on the $(s, t)$-coordinate then $\mathbb{R}^{\mathbf{Z}^{2}}$ is partially ordered by:

$$
x \leq y \quad \Leftrightarrow \quad \pi_{s, t}(x) \leq \pi_{s, t}(y) \text { for all }(s, t) .
$$

For two probability measures $P^{\prime}, P^{\prime \prime}$ on the Borel $\sigma$-algebra of $\mathbb{R}^{Z^{2}}$ we say that $P^{\prime}$ is stochastically smaller than or equal to $P^{\prime \prime}\left(P^{\prime}<P^{\prime \prime}\right)$ if

$$
\int \varphi d P^{\prime} \leq \int \varphi d P^{\prime \prime}
$$

holds for every measurable increasing function $\varphi: \mathbb{R}^{\mathbf{Z}^{2}} \rightarrow \mathbb{R}$ integrable with respect to $P^{\prime}$ and $P^{\prime \prime}$. We define the measurable transformation $T$ on the subspace $E=\mathbb{R}^{\mathcal{N}}$ by

$$
\pi_{s, t} \circ T=\pi_{s+1, t+1} \quad \text { for all }(s, t) \in \mathcal{N} .
$$

A family $X=\left\{X_{s, t}:(s, t) \in \mathcal{N}\right\}$ of real valued random variables on a probability space $(\Omega, \mathscr{A}, P)$ is called a subadditive superstationary process if

(I) $X_{s, t} \leq X_{s, r}+X_{r, t} \quad$ for all $(s, r),(r, t) \in \mathcal{N}$;

(II) $P_{X}>P_{T X}$;

where $P_{X}, P_{T X}$ denote the probabilities on $E$ induced by $X$, respectively $T \circ X$. 
Before starting our investigations let us first have a look at the following

Proposition 1. Let $\left\{x_{s, t}:(s, t) \in \mathcal{N}\right\}$ be a subadditive family of real numbers (i.e. $x_{s, t} \leq x_{s, r}+x_{r, t}$ for all $\left.(s, r),(r, t) \in \mathcal{N}\right)$. For $a<b \in \mathbb{R}, N \in \mathbb{N}$, let $n$ be a positive integer such that there exist integers

$$
0 \leq u_{1}<v_{1}<\cdots<u_{n}<v_{n} \leq N
$$

satisfying

$$
\begin{aligned}
v_{i} b-u_{i} a & \leq x_{u_{i}, v_{i}} & & i \leq n, \\
x_{v_{i}, u_{i+1}} & \leq u_{i+1} a-v_{i} b & & i \leq n-1 .
\end{aligned}
$$

Call a finite or possibly empty set $R=\left\{s_{1}, t_{1}, \ldots s_{m_{R}}, t_{m_{R}}\right\}$ of integers admissible if

$$
0 \leq s_{1}<t_{1} \leq s_{2}<t_{2} \leq \cdots \leq s_{m_{R}}<t_{m_{R}} \leq N \text {. }
$$

For each admissible $R$ consider

$$
\begin{array}{ll}
S_{R}=\sum_{i=1}^{m_{R}} x_{s_{i}, t_{i}}-b t_{i}+a s_{i} & m_{R}>0 \\
S_{R}=0 & m_{R}=0 .
\end{array}
$$

Under these assumptions there exists an admissible $Q$ with

$$
S_{Q} \geq S_{R} \text { and } m_{Q} \geq n \text {. }
$$

Proof. For $m_{R} \geq n$ the assertion is trivial. Therefore it suffices to show that for $m_{R}<n$ we can find an admissible $Q$ with $S_{Q} \geq S_{R}$ and $m_{Q}=m_{R}+1$.

Let $s_{m_{R}+1}=N$. Now $m_{R}+1 \leq n$ holds, thus $v_{m_{R}+1}$ is well defined and we have $v_{m_{R}+1} \leq N=s_{m_{R}+1}$. Let $i$ be the smallest integer satisfying $v_{i} \leq s_{i}$. If $i=1$, choose

$$
Q=\left\{u_{1}, v_{1}, s_{1}, t_{1}, \ldots, s_{m_{R}}, t_{m_{R}}\right\} .
$$

$Q$ is admissible and $m_{Q}=m_{R}+1$.

$$
S_{Q}=S_{R}+\left(x_{u_{1}, v_{1}}-b v_{1}+a u_{1}\right) \geq S_{R}
$$

by assumption. We can now assume $i>1$ :

By definition of $i, s_{i-1}<v_{i-1}<u_{i}<v_{i} \leq s_{i}$. If $u_{i} \geq t_{i-1}$, choose

$$
Q=\left\{s_{1}, t_{1}, \ldots, s_{i-1}, t_{i-1}, u_{i}, v_{i}, s_{i}, t_{i}, \ldots, s_{m_{R}}, t_{m_{R}}\right\} .
$$

(Of course $Q$ ends with $v_{i}$ if $i=m_{R}+1$ ). $Q$ is admissible and $m_{Q}=m_{R}+1$. Again

$$
S_{Q}=S_{R}+\left(x_{u_{i}, v_{i}}-b v_{i}+a u_{i}\right) \geq S_{R}
$$

by assumption. It remains to consider the case $i>1, u_{i}<t_{i-1}$ : Choose

$$
Q=\left\{s_{1}, t_{1}, \ldots, s_{i-2}, t_{i-2}, s_{i-1}, v_{i-1}, u_{i}, t_{i-1}, s_{i}, t_{i}, \ldots, s_{m_{R}}, t_{m_{R}}\right\} .
$$

$Q$ is admissible and $m_{Q}=m_{R}+1$.

$$
\begin{aligned}
S_{Q}=S_{R} & -\left(x_{s_{i-1}, t_{i-1}}-b t_{i-1}+a s_{i-1}\right) \\
& +\left(x_{s_{i-1}, v_{i-1}}-b v_{i-1}+a s_{i-1}\right) \\
& +\left(x_{u_{i}, t_{i-1}}-b t_{i-1}+a u_{i}\right) \\
=S_{R} & +\left(x_{s_{i-1}, v_{i-1}}+x_{u_{i}, t_{i-1}}-x_{s_{i-1}, t_{i-1}}\right)-b v_{i-1}+a u_{i}
\end{aligned}
$$




$$
\begin{aligned}
\geq & S_{R}+\left(x_{s_{i-1}, v_{i-1}}+x_{u_{i}, t_{i-1}}-\left[x_{s_{i-1}, v_{i-1}}+x_{v_{i-1}, u_{i}}+x_{u_{i}, t_{i-1}}\right]\right) \\
& \quad-b v_{i-1}+a u_{i} \\
= & S_{R}+\left(a u_{i}-b v_{i-1}-x_{v_{i-1}, u_{i}}\right) \geq S_{R}
\end{aligned}
$$

by assumption.

THEOREM 1 (Upcrossing inequality for discrete time). Let $X=\left\{X_{s, t}\right\}$ be a subadditive superstationary process. For $N \in \mathbb{N}, a<b \in \mathbb{R}$ and $x \in E$ define the 'number of upcrossings till $N^{\prime}$ by

$$
\begin{gathered}
W_{N}(x)=\max \left\{m: \exists 0 \leq u_{1}<v_{1}<\cdots<u_{m}<v_{m} \leq N\right. \text { such that } \\
\pi_{u_{i}, v_{i}}(x) \geq b v_{i}-a u_{i} \quad i \leq m, \quad \text { and } \\
\left.\pi_{v_{i}, u_{i+1}}(x) \leq a u_{i+1}-b v_{i} \quad i \leq m-1\right\} .
\end{gathered}
$$

If $X_{0,1}^{+}$is integrable with respect to $P$ then

$$
\int W_{N} \circ X d P \leq(b-a)^{-1} \int\left(X_{0,1}-a\right)^{+} d P .
$$

Proof. As only finitely many functions are involved, $W_{N}$ and $W_{N} \circ X$ are measurable. We want to use proposition 1 . Therefore we define, for each admissible sequence $R=\left\{s_{1}, t_{1}, \ldots, s_{m_{R}}, t_{m_{R}}\right\}$ and each $x \in E$,

$$
S_{R}(x)=\sum_{i=1}^{m_{R}} \pi_{s_{i}, t_{i}}(x)-b t_{i}+a s_{i}
$$

Let $M$ be the maximum of the $S_{R}$ taken over all admissible $R$. $m_{R}=0$ implies $S_{R} \equiv 0$. Hence $M$ is a positive function and there exists a constant $C(a, b)<\infty$ so that

$$
M \leq \sum_{0 \leq s<t \leq N} \pi_{s, t}^{+}+C(a, b)
$$

$X_{0,1}^{+}$is integrable with respect to $P$, hence $\pi_{0,1}^{+}$is integrable with respect to $P_{X}$. As all projections are increasing, we see that all $S_{R}$ are increasing. By subadditivity and superstationarity we conclude that all $\pi_{s, t}^{+}$are integrable. Thus, $M$ is an increasing function and integrable with respect to both $P_{X}$ and $P_{T X}$. We want to show that

$$
\left(\pi_{0,1}(x)-a\right)^{+}-(b-a) W_{N}(x) \geq M(x)-M \circ T(x)
$$

holds with probability one (both for $P_{X}$ and $P_{T X}$ ). Let $R=\left\{0 \leq s_{1}<t_{1} \leq \cdots \leq\right.$ $s_{m_{R}}<t_{m_{R}}$ be admissible. Assume $s_{1}>0$. Then

$$
\begin{aligned}
S_{R}(x) & =\sum_{i=1}^{m_{R}}\left(\pi_{s_{i}, t_{i}}(x)-b t_{i}+a s_{i}\right) \\
& =\sum_{i=1}^{m_{R}}\left[\pi_{s_{i}-1, t_{i}-1}(T x)-b\left(t_{i}-1\right)+a\left(s_{i}-1\right)-(b-a)\right] \\
& =\sum_{i=1}^{m_{R}}\left[\pi_{s_{i}-1, t_{i}-1}(T x)-b\left(t_{i}-1\right)+a\left(s_{i}-1\right)\right]-m_{R}(b-a) .
\end{aligned}
$$


$R^{\prime}=\left\{0 \leq s_{1}-1<t_{1}-1 \leq \cdots \leq s_{m_{R}}-1<t_{m_{R}}-1 \leq N\right\}$ is admissible. Hence

$$
\begin{aligned}
S_{R}(x) & =S_{R^{\prime}}(x)-m_{R}(b-a) \\
& \leq M \circ T(x)-m_{R}(b-a)+\left(\pi_{0,1}(x)-a\right)^{+} .
\end{aligned}
$$

The case $s_{1}=0$ will be split into two subcases. We have

$$
\begin{aligned}
S_{R}(x) & =\sum_{i=1}^{m_{R}}\left(\pi_{s_{i}, t_{i}}(x)-b t_{i}+a s_{i}\right) \\
& =\pi_{0, t_{1}}(x)-b t_{1}-m_{R}(b-a)+\sum_{i=2}^{m_{R}}\left[\pi_{s_{i}-1, t_{i}-1}(T x)-b\left(t_{i}-1\right)+a\left(s_{i}-1\right) .\right.
\end{aligned}
$$

First assume $t_{1}>1$. Let $s_{1}^{\prime}=1$. Because of assumption (I) we can find a set $A \subset E$ with $P_{X}(A)=P_{T X}(A)=1$ such that $\pi_{s, t}(x) \leq \pi_{s, r}(x)+\pi_{r, t}(x)$ holds for all $x \in A$ and all $(s, r),(r, t) \in \mathcal{N}$. Let $x \in A$, then

$$
\begin{aligned}
S_{R}(x) \leq & \pi_{0,1}(x)+\pi_{1, t_{1}}(x)-b t_{1}-\left(m_{R}-1\right)(b-a)+\sum_{i=2}^{m_{R}}[\cdots] \\
= & \pi_{0,1}(x)-a+\left[\pi_{s_{1}^{\prime}-1, t_{1}-1}(T x)-b\left(t_{1}-1\right)+a\left(s_{1}^{\prime}-1\right)\right] \\
& -m_{R}(b-a)+\sum_{i=2}^{m_{R}}[\cdots] .
\end{aligned}
$$

$R^{\prime}=\left\{0 \leq s_{1}^{\prime}-1<t_{1}-1 \leq \cdots \leq s_{m_{R}}-1<t_{m_{R}}-1 \leq N\right\}$ is admissible. Hence

$$
\begin{aligned}
S_{R}(x) & \leq\left(\pi_{0,1}(x)-a\right)-m_{R}(b-a)+S_{R^{\prime}}(T x) \\
& \leq M \circ T(x)-m_{R}(b-a)+\left(\pi_{0,1}(x)-a\right)^{+} .
\end{aligned}
$$

It remains to consider the case $t_{1}=1$. Then

$$
\begin{aligned}
S_{R}(x) & =\pi_{0,1}(x)-b-\left(m_{R}-1\right)(b-a)+\sum_{i=2}^{m_{R}}[\cdots] \\
& =\pi_{0,1}(x)-a-m_{R}(b-a)+\sum_{i=2}^{m_{R}}[\cdots] .
\end{aligned}
$$

$R^{\prime}=\left\{0 \leq s_{2}-1<t_{2}-1 \leq \cdots \leq s_{m_{R}}-1<t_{m_{R}}-1 \leq N\right\}$ is admissible. Hence

$$
\begin{aligned}
S_{R}(x) & =\left(\pi_{0,1}(x)-a\right)-m_{R}(b-a)-S_{R^{\prime}}(T x) \\
& \leq M \circ T(x)-m_{R}(b-a)+\left(\pi_{0,1}(x)-a\right)^{+} .
\end{aligned}
$$

Thus, we have

$$
S_{R}(x) \leq M \circ T(x)-m_{R}(b-a)+\left(\pi_{0,1}(x)-a\right)^{+}
$$

for each admissible $R$ and $x \in A$. Using proposition 1 we can find an admissible $Q$ satisfying $S_{Q}(x) \geq S_{R}(x)$ and $m_{Q} \geq W_{N}(x)$. Therefore

$$
\begin{aligned}
S_{R}(x) \leq S_{Q}(x) & \leq\left(\pi_{0,1}(x)-a\right)^{+}-m_{Q}(b-a)+M \circ T(x) \\
& \leq\left(\pi_{0,1}(x)-a\right)^{+}-W_{N}(x)(b-a)+M \circ T(x) .
\end{aligned}
$$

Since this holds for each admissible $R$ we obtain

$$
M(x) \leq\left(\pi_{0,1}(x)-a\right)^{+}-W_{N}(x)(b-a)+M \circ T(x)
$$


which yields (1.1). Now we can conclude

$$
\begin{aligned}
(b-a) \int W_{N} \circ X d P= & (b-a) \int W_{N}(x) d P_{X}(x) \\
\leq & \int M \circ T(x)-M(x)+\left(\pi_{0,1}(x)-a\right)^{+} d P_{X}(x) \\
= & \int M \circ T(x) d P_{X}(x)-\int M(x) d P_{X}(x) \\
& +\int\left(\pi_{0,1}(x)-a\right)^{+} d P_{X}(x) \\
= & \int M(x) d P_{T X}(x)-\int M(x) d P_{X}(x)+\int\left(X_{0,1}-a\right)^{+} d P \\
\leq & \int\left(X_{0,1}-a\right)^{+} d P,
\end{aligned}
$$

because $M$ is an increasing measurable function and integrable with respect to both $P_{X}$ and $P_{T X}$. Rearranging this inequality will complete our proof.

Let us now turn to the case of continuous time. Our new parameter set is

$$
\mathcal{N}_{c}=\left\{(s, t) \in \mathbb{R}^{2}: 0 \leq s<t\right\} .
$$

If $X=\left\{X_{s, t}:(s, t) \in \mathcal{N}_{c}\right\}$ is a sequence of real valued random variables on $(\Omega, \mathscr{A}, P)$ and $\left(s_{1}, t_{1}\right), \ldots,\left(s_{n}, t_{n}\right) \in \mathcal{N}_{c}$ then $\left(X_{s_{1}, t_{1}}, \ldots, X_{s_{n}, t_{n}}\right) P$ denotes the probability on $\mathbb{R}^{n}$ induced by the random vector $\left(X_{s_{1}, t_{1}}, \ldots, X_{s_{n}, t_{n}}\right) . X$ is called a continuous parameter subadditive superstationary process if

$\operatorname{cp}(\mathrm{I}) \quad X_{s, t} \leq X_{s, r}+X_{r, t}$, for all $(s, r),(r, t) \in \mathcal{N}_{c} ;$ and

$\operatorname{cp}$ (II) $\left.\left(X_{s_{1}, t_{1}}\right), \ldots, X_{s_{n}, t_{n}}\right) P>\left(X_{s_{1}+u, t_{1}+u}, \ldots, X_{s_{n}+u, t_{n}+u}\right) P$, for all $\left(s_{1}, t_{1}\right), \ldots$, $\left(s_{n}, t_{n}\right) \in \mathcal{N}_{c}$ and all $u>0$.

(Additional integrability conditions used by Hachem will not be part of our definition.)

For fixed $\zeta>0$ and $a<b \in \mathbb{R}$ define the 'number of upcrossings till $\zeta$ ' as

$$
\begin{aligned}
& W_{6}(\omega)=\max \left\{m: \exists 0 \leq s_{1}<t_{1}<\cdots<s_{m}<t_{m}<\zeta\right. \text { such that } \\
& \qquad \begin{aligned}
X_{s_{i}, t_{i}}(\omega) & >t_{i} b-s_{i} a, \quad i \leq m \\
\text { and } \quad X_{t_{i}, s_{i+1}}(\omega) & \left.<s_{i+1} a-t_{i} b, \quad i \leq m-1\right\}
\end{aligned}
\end{aligned}
$$

Convergence of the averages $t^{-1} X_{0,4}$ was proved by Hachem under two assumptions: separability and integrability of the maximum oscillation, (i.e. $E\left(\sup _{0 \leq s<t<1} X_{s, t}^{+}\right)<$ $+\infty)$.

We will now give an example which shows that these assumptions are not sufficient for a process to have an integrable number of upcrossings.

Example. Let $\nu_{0}, \nu_{1}, \ldots$ be i.i.d. random variables such that $\nu_{k}$ attaches mass $(n(n+1))^{-1}$ to $n^{2} 2 \pi$, let $\eta$ be uniformly distributed on $[0,1]$ and independent of 
the $\nu_{k}$. Define

$$
\hat{X}_{t}=\sqrt[4]{\nu_{n}(2 \pi)^{-1}} \sin \left(\nu_{n}(t-n)\right) \quad \text { for } 0 \leq n<t<n+1
$$

and

$$
X_{t}=\hat{X}_{t+\eta}
$$

$\left(X_{t}\right)$ is a stationary process with continuous sample functions and $E\left(X_{t}\right)<\infty$ holds. Furthermore, for $0 \leq n<t<n+1$ :

$$
X_{t}^{+} \leq \sqrt[4]{\nu_{n}(2 \pi)^{-1}}+\sqrt[4]{\nu_{n+1}(2 \pi)^{-1}}
$$

which yields

$$
E\left(\sup _{t \in[0,1]} X_{t}^{+}\right)<\infty \text {. }
$$

Let $0 \leq n$ be an integer and $0 \leq d<1$. Consider the interval $(n, n+d)$. Choose an integer $m$ such that $\sqrt{m}>n+1$. For $\omega \in A_{m}=\left\{\nu_{n}=m^{2} 2 \pi\right\} \cap\left\{\nu_{n+1} \geq m^{2} 2 \pi\right\}$ it is easily seen that if $t$ moves from $n$ to $n+d$, the process $\left(X_{t}\right)$ has about $d m^{2}$ upcrossings of the interval $(-(n+1),+(n+1))$. (Note that $t<n+1$.) For $\omega \in A_{m+1}=$ $\left\{\nu_{n}=(m+1)^{2} 2 \pi\right\} \cap\left\{\nu_{n+1} \geq(m+1)^{2} 2 \pi\right\}$ there are about $d(m+1)^{2}$ upcrossings. Now all $A_{k}$ for $k \geq m$ are disjoint. By independence we conclude that

$$
\begin{aligned}
P\left(A_{k}\right) & =P\left(\nu_{n}=k^{2} 2 \pi\right) P\left(\nu_{n+1} \geq k^{2} 2 \pi\right) \\
& =(k(k+1))^{-1} \sum_{j=k}^{\infty}(j(j+1))^{-1} \\
& \geq k^{-2}(k+1)^{-1} .
\end{aligned}
$$

Hence we have at least about $d k^{2}$ upcrossings on a set of probability greater than $k^{-2}(k+1)^{-1}$. But $\sum_{k=m}^{\infty} d k^{2} k^{-2}(k+1)^{-1}=\infty$ and thus the number of upcrossings is not integrable. Because of stationarity the same holds for an arbitrary interval contained in $\mathbb{R}^{+}$. Finally define $X_{s, t}=X_{t}-X_{s}$. This process is clearly subadditive superstationary and has at least as many upcrossings as the process $\left(X_{t}\right)$. As shown above new assumptions will be necessary in order to prove an upcrossings inequality.

Let $f: \mathcal{N}_{c} \rightarrow \mathbb{R}$. We say $f$ is continuous from above at $\left(s_{0}, t_{0}\right) \in \mathcal{N}_{c}$ if for a sequence $\left(s_{n}, t_{n}\right)$ satisfying $\left(s_{n}, t_{n}\right) \geq\left(s_{0}, t_{0}\right)$ for all $n$ and $\left(s_{n}, t_{n}\right) \rightarrow\left(s_{0}, t_{0}\right)$ we have

$$
f\left(s_{n}, t_{n}\right) \rightarrow f\left(s_{0}, t_{0}\right) \text {. }
$$

$f$ is called continuous from above if it is continuous from above at each $(s, t) \in \mathcal{N}_{c}$ Here is our upcrossing inequality:

Theorem 2 (Upcrossing inequality for continuous time). Let $X=\left\{X_{s, t}:(s, t) \in \mathcal{N}_{c}\right\}$ be a continuous parameter subadditive superstationary process and for $\zeta>0, a<b \in \mathbb{R}$ let $W_{\zeta}$ be defined as above. If

(2.1) $\sup t^{-1} E X_{0, t}^{+}<\infty$ holds, and

(2.2) $X$ has sample functions that are continuous from above, then

$$
\int W_{\zeta} d P \leq(b-a)^{-1}\left(\sup t^{-1} E X_{0, t}^{+}+|a|\right) \text {. }
$$


Proof. It is easily seen that for an integer $k$ the sequence

$$
X^{k}=\left\{X_{m, n}^{k}=2^{k} X_{m 2^{-k}, n 2^{-k}}: 0 \leq m<n \in \mathbb{N}\right\}
$$

forms a subadditive superstationary process. Let $N(k)$ be the largest integer smaller than or equal to $2^{k} \zeta$ and denote by $W_{N(k)}^{k}$ the number of upcrossings till $N(k)-1$ of the process $X^{k}$. Applying theorem 1 we obtain

$$
\begin{aligned}
(b-a) \int W_{N(k)}^{k} d P & \leq \int\left(X_{0,1}^{k}-a\right)^{+} d P \\
& =\int\left(2^{k} X_{\left.0,2^{-k}-a\right)^{+} d P}\right. \\
& \leq \sup t^{-1} E X_{0, t}^{+}+|a| .
\end{aligned}
$$

We want to show that $W_{N(k)}^{k}$ increases pointwise to $W_{\zeta}$ as $k$ tends to $\infty$. Of course $W_{N(k)}^{k} \leq W_{N(k+1)}^{k+1} \leq W_{\zeta}$ holds for all $k$. Consider a sequence $0 \leq s_{1}<t_{1}<\cdots<s_{n}<$ $t_{n}<\zeta$ such that for fixed $\omega \in \Omega$,

$$
\begin{aligned}
t_{i} b-s_{i} a & <X_{s_{i}, t_{i}}(\omega), & & 1 \leq i \leq n, \\
X_{t_{i}, s_{i+1}}(\omega) & <s_{i+1} a-t_{i} b, & & 1 \leq i \leq n-1 .
\end{aligned}
$$

Choose, in $\bigcup_{k=1}^{\infty} \mathbb{N} 2^{-k}$, sequences $s_{i}^{m} \rightarrow s_{i},\left(s_{i}^{m} \geq s_{i}\right), t_{i}^{m} \rightarrow t_{i},\left(t_{i}^{m} \geq t_{i}\right)$, satisfying $0 \leq$ $s_{1}^{m}<t_{1}^{m}<\cdots<s_{n}^{m}<t_{n}^{m}<\zeta$ for all $m \in \mathbb{N}$. Because of continuity from above we can find an integer $m_{0}$ such that for all $m \geq m_{0}$

$$
\begin{aligned}
X_{s_{i}^{m}, t_{i}^{m}}(\omega) & >t_{i}^{m} b-s_{i}^{m} a, & & 1 \leq i \leq n, \\
X_{t_{i}^{m}, s_{i+1}^{m}}(\omega) & <s_{i+1}^{m} a-t_{i}^{m} b, & & 1 \leq i \leq n-1 .
\end{aligned}
$$

Let $k_{0}=\min \left\{k \in \mathbb{N}: 2^{k} t_{i}^{m_{0}} \in \mathbb{N}, 2^{k} s_{i}^{m_{0}} \in \mathbb{N}\right.$ for all $\left.1 \leq i \leq n\right\}$. Then, of course,

$$
W_{N\left(k_{0}\right)}^{k_{0}}(\omega) \geq n \text {. }
$$

Hence $W_{N(k)}^{k}$ increases to $W_{\xi}$. By the monotone convergence theorem we obtain

$$
\int W_{\zeta} d P \leq(b-a)^{-1}\left(\sup t^{-1} E X_{0, t}^{+}+|a|\right) \text {. }
$$

Remarks. If $\left(X_{t}\right)_{t \geq 0}$ is a continuous parameter superstationary process (i.e. $\left(X_{t_{1}}, \ldots, X_{t_{n}}\right) P>\left(X_{t_{1}+u}, \ldots, X_{t_{n}+u}\right) P$ for all $0 \leq t_{1}<\cdots<t_{n}$ and $\left.u>0\right)$ with sample functions that are Riemann-integrable on each interval $[0, a] \subset \mathbb{R}$ then some arguments in [4] can be used to show that the sequence $X_{s, t}=\int_{s}^{t} X_{\nu} d \nu$ forms a continuous time subadditive superstationary process. Furthermore, if $X_{0}^{+}$is integrable then $E X_{0}^{+}=\sup t^{-1} E X_{0, t}^{+}$and both assumptions in theorem 2 are satisfied.

The author wishes to thank Prof. Dr. U. Krengel for his support and encouragement.

\section{REFERENCES}

[1] M. Abid. Un théorème ergodique pour des processus sousadditifs et sur-stationnaires. C.R. Acad. Sc. Paris, 287, A (1978), 149-152.

[2] E. Bishop. Foundations of Constructive Analysis. McGraw Hill: New York.

[3] B. Hachem. Sur le théorème ergodique sur-stationnaire et sous-additif dans $L^{p}(1 \leq p<\infty)$. C.R. Acad. Sc. Paris 292, I (1981), 837-840. 
[4] T. Kamae, U. Krengel \& G. O'Brien. Stochastic inequalities in partially ordered spaces. Ann. of Prob. 5, no. 6 (1977), 899-912.

[5] J. F. C. Kingman. Subadditive ergodic theory. Ann. of Prob. 1, 883-909.

[6] U. Krengel. Un théorème ergodique pour les processus sur-stationnaires. C.R. Acad. Sc. Paris. 282, A (1976), 1019-1021.

[7] M. D. Smeltzer. The subadditive ergodic theorem. Ph.D. Thesis, Yale University, 1976. 